\title{
Energy balance of a laser ablation plume expanding in a background gas
}

\section{Amoruso, Salvatore; Schou, Jørgen; Lunney, James G.}

\section{Published in:}

Applied Physics A: Materials Science \& Processing

Link to article, DOI:

10.1007/s00339-010-5753-6

Publication date:

2010

Document Version

Publisher's PDF, also known as Version of record

Link back to DTU Orbit

Citation (APA):

Amoruso, S., Schou, J., \& Lunney, J. G. (2010). Energy balance of a laser ablation plume expanding in a background gas. Applied Physics A: Materials Science \& Processing, 101(1), 209-214.

https://doi.org/10.1007/s00339-010-5753-6

\section{General rights}

Copyright and moral rights for the publications made accessible in the public portal are retained by the authors and/or other copyright owners and it is a condition of accessing publications that users recognise and abide by the legal requirements associated with these rights.

- Users may download and print one copy of any publication from the public portal for the purpose of private study or research.

- You may not further distribute the material or use it for any profit-making activity or commercial gain

- You may freely distribute the URL identifying the publication in the public portal 


\title{
Energy balance of a laser ablation plume expanding in a background gas
}

\author{
Salvatore Amoruso · Jørgen Schou • James G. Lunney
}

Received: 31 October 2009 / Accepted: 27 April 2010 / Published online: 29 May 2010

(C) Springer-Verlag 2010

\begin{abstract}
The energy balance of a laser ablation plume in an ambient gas for nanosecond pulses has been investigated on the basis of the model of Predtechensky and Mayorov (PM), which provides a relatively simple and clear description of the essential hydrodynamics. This approach also leads to an insightful description in dimensionless units of how the initial kinetic energy of the plume is dissipated into kinetic and thermal energy of the background gas. Eventually when the plume has stopped, the initial kinetic energy of the plume is converted into thermal energy of the plume and background gas.
\end{abstract}

\section{Introduction}

Pulsed laser deposition (PLD) and laser-generated nanoparticle formation often take place in a background gas [1, 2]. Even though these techniques have been applied extensively for a number of years, there is no complete picture of the dynamics of a laser ablation plume in a background gas. PLD of a film by nanosecond lasers is an extremely complex process, which runs through several stages from ini-

\section{S. Amoruso}

Coherentia CNR SPIN and Dipartimento di Scienze Fisiche,

Università degli Studi di Napoli Federico II, Complesso

Universitario di Monte S. Angelo, Via Cintia, 80126 Napoli, Italy

J. Schou $(\bowtie)$

Department of Photonics Engineering, Ris $\emptyset$ Campus, Technical

University of Denmark, 4000 Roskilde, Denmark

e-mail: josc@fotonik.dtu.dk

Fax: +45-467-74565

J.G. Lunney

School of Physics, Trinity College Dublin, Dublin 2, Ireland tial laser irradiation of a solid target, material ejection and transfer through vacuum or a background gas to a substrate [3-5]. A more comprehensive knowledge of plume dynamics in a background gas is clearly desirable, in particular because most of the existing treatments are limited to one or two target materials into a single background gas [6-9]. The expansion of a silicon ablation plume in helium and argon gases has been modeled quite comprehensively, but the results are difficult to apply to other plume-background gas systems [8].

In the present work we have applied the gas-dynamical model of Predtechensky and Mayorov (PM) [10] for the silver ablation plume in different gases. The work is partly a continuation of previous work on the expansion of a plume $[11,12]$ and partly a new analysis of the energy balance of the plume expansion. In the PM-model all plume material is located in a thin, expanding spherical shell which sweeps up the background gas on a thin shell on the outer surface of the plume shell [11]. Both shells expand against the pressure of the background gas (Fig. 1). Eventually, the increased mass of the swept-up gas and the plume mass lead to a deceleration of the contact front.

As demonstrated in Refs. [11, 12] the PM-model can be solved analytically such that, for example, the stopping range can be determined. The PM-model can also be utilized to describe the distribution of energy in thermal and kinetic components of the plume in a simple manner and shows quite good agreement with the analysis by Arnold et al. [9].

\section{The theoretical model}

At the termination of the laser pulse the plume energy is predominantly thermal, but the energy is rapidly converted to 


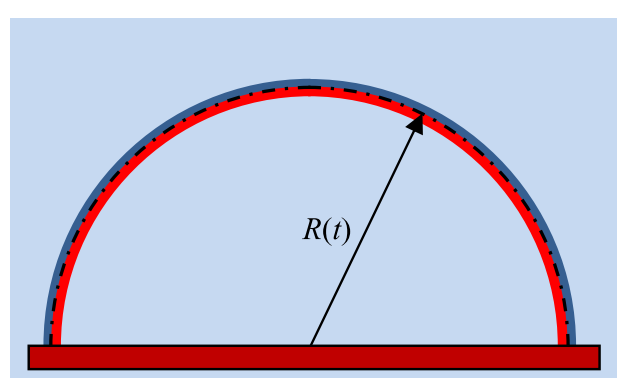

Fig. 1 Geometry of the plume expansion in the PM-model. Red color: target and plume material at the radius $R(t)$. Light blue color: background gas. Dark blue: swept-up background gas on the shell of radius $R(t)$

kinetic energy during the early stage expansion. The main assumptions for the PM-model, that the initial plume energy is all kinetic and that the expansion is 3-dimensional after a short, initial 1-dimensional expansion, are largely fulfilled [11].

The momentum equation for an infinitesimally thin, hemispherical gas layer at $R$ containing all the mass of the plume as well as the swept-up gas (Fig. 1) is

$\frac{d}{d t}\left[\left(M_{p}+\frac{2}{3} \pi \rho_{g} R^{3}\right) u\right]=-2 \pi R^{2} p_{g}$,

where $p_{g}$ is the gas pressure, $\rho_{g}$ is the gas density, $M_{p}$ the mass of the plume and the radial velocity of the plume/gas layer at time $t$ is $u(t)=d R / d t$. The equation can be solved analytically with the change of variables, $d / d t=$ $u(R) d / d R$ as in [10], but here we express (1) in terms of dimensionless variables [11]:

$\frac{d}{d \tau}\left[\left(1+\xi^{3}\right) \eta\right]=-3 \xi^{2}$

where the dimensionless plume front position is

$\xi=A^{-1 / 3} R$

the dimensionless time is

$\tau=A^{-1 / 3} c_{g} t$

and the dimensionless velocity is

$\eta=d \xi / d \tau=1 / c_{g}(d R / d t)=u / c_{g}$

The initial, dimensionless velocity is then

$\eta_{0}=u_{0} / c_{g}$

where $u_{0}=u(t=0)$ is the initial plume front velocity. The characteristic velocity $c_{g}=\sqrt{k_{B} T / m_{g}}\left(k_{B}\right.$ is Boltzmann's constant, $T$ is the ambient gas temperature and $m_{g}$ the atomic/molecular mass of background gas). The parameter $A$, which is given by

$A=3 M_{p} /\left(2 \pi \rho_{g}\right)$,

is determined by the condition that the mass of displaced gas is equal to the mass of the ablation plume. Equation (2) has an analytical solution which accounts fairly well for the expansion from nearly-free propagation of the plume close to the target, through a regime of point-blast-wave behavior, to the point where the plume comes to a complete halt. The dimensionless position as a function of time agrees well with experimental data for silver ions expanding in argon, oxygen and xenon gas [11]. The model has also provided a good description of the influence of substrate temperature on the plume behavior for complex oxides in an oxygen atmosphere [12-14]. Results with a PM-model modified to a 1-dimensional expansion were also obtained for nanoparticles produced by fs-laser ablation [15].

\section{Energy balance of the plume expansion}

Since we have assumed that the thermal energy at the initial expansion is negligible, the kinetic energy of the shell with the plume mass $M_{p}$ at the start of the expansion and at time $t$ with a velocity $u_{0}$, respectively, is

$E_{0}=\frac{1}{2} M_{p} u_{0}^{2}$

and

$E_{p, \mathrm{k}}(t)=\frac{1}{2} M_{p} u^{2}(t)$

The kinetic energy of the swept-up gas at time $t$ is

$$
\begin{aligned}
E_{g, \mathrm{k}}(t) & =\frac{1}{2}\left(\int_{0}^{R(t)} 2 \pi r^{2} \rho_{g} \mathrm{~d} r\right) u^{2}(t) \\
& =\frac{1}{3} \pi \rho_{g} R^{3}(t) u^{2}(t) .
\end{aligned}
$$

For the final energy $E_{\text {tot }}$ at time $t$ we obtain

$E_{\mathrm{tot}}(t)=E_{p, \mathrm{k}}(t)+E_{g, \mathrm{k}}(t)+E_{p+g, \mathrm{th}}(t)$,

such that the energy is the sum of the kinetic energy of the plume, $E_{p, \mathrm{k}}$, the kinetic energy of the swept-up gas, $E_{g, \mathrm{k}}$ and the internal, thermal energy of the plume and the sweptup gas $E_{p+g \text {,th. }}$.

The initial energy, $E_{\text {in }}$, of the system is the sum of the initial energy of the plume, $E_{0}$, and the internal energy $E_{g}$,int, which initially is stored in that part of the background gas (at 
rest) which has been swept up during the plume expansion up to time $t$ :

$E_{\text {in }}=E_{0}+E_{g, \text { int }}(R(t))$,

$E_{g, \text { int }}(R(t))=\int \frac{p_{g} d V}{\gamma_{g}-1}=\frac{\frac{2}{3} \pi p_{g} R^{3}(t)}{\gamma_{g}-1}$

where $\gamma_{g}$ is the adiabatic constant of the background gas.

For the total energy balance we now need the work, $L_{\text {ext }}$, made by the external pressure during the expansion:

$L_{\mathrm{ext}}(t)=-\int_{0}^{R(t)} 2 \pi r^{2} p_{g} d r=-\frac{2}{3} \pi p_{g} R^{3}(t)$.

Since the extra energy of the system at time $t$ originates from the external work on the system, the total energy balance is thus

$E_{\text {fin }}(t)-E_{\text {in }}=L_{\text {ext }}(t)$.

We will now for simplicity convert the energies to dimensionless quantities by dividing all energies with the initial plume energy $E_{0}$ such that

$\varepsilon_{0}=E_{0} / E_{0}=1$,

the dimensionless, kinetic energy of the plume at time $\tau$

$\varepsilon_{p, \mathrm{k}}(t)=E_{p, \mathrm{k}} / E_{0}=\frac{\eta^{2}(\tau)}{\eta_{0}^{2}}$,

the dimensionless, kinetic energy of the swept-up gas

$\varepsilon_{g, \mathrm{k}}(t)=E_{g, \mathrm{k}} / E_{0}=\xi^{3}(\tau) \frac{\eta^{2}(\tau)}{\eta_{0}^{2}}$,

the dimensionless, internal energy of the swept-up gas

$\varepsilon_{g, \text { int }}(\tau)=E_{g, \text { int }} / E_{0}=\frac{2}{\left(\gamma_{g}-1\right)} \frac{\xi^{3}}{\eta_{0}^{2}}$

and finally dimensionless work made by the external pressure

$\Lambda_{\mathrm{ext}}=L_{\mathrm{ext}} / E_{0}=-2 \frac{\xi^{3}}{\eta_{0}^{2}}$.

The energy balance expressed in dimensionless units is now

$\frac{E_{\text {fin }}(t)-E_{\text {in }}}{E_{0}}=\varepsilon_{\text {fin }}-\varepsilon_{\text {in }}=\Lambda_{\text {ext }}$,

which can be brought into the following form:

$$
\begin{aligned}
& {\left[\varepsilon_{p, \mathrm{k}}(\tau)+\varepsilon_{g, \mathrm{k}}(\tau)+\varepsilon_{p+g, \mathrm{th}}(\tau)\right]-\left[1+\varepsilon_{g, \mathrm{int}}(\tau)\right]} \\
& \quad=\Lambda_{\mathrm{ext}}(\tau)
\end{aligned}
$$

such that the excess thermal energy can be determined as

$$
\begin{aligned}
\varepsilon_{\mathrm{th}} & =\varepsilon_{p+g, \mathrm{th}}(\tau)-\varepsilon_{g, \text { int }}(\tau) \\
& =1-\left(1+\xi^{3}(\tau)\right) \frac{\eta^{2}(\tau)}{\eta_{0}^{2}}-2 \frac{\xi^{3}}{\eta_{0}^{2}} .
\end{aligned}
$$

These equations provide us with the kinetic energy of the plume (see (12b)), the kinetic energy of the swept-up gas (see (12c)) as well as the total excess thermal energy of the plume and swept-up gas (see (15)) as a function of time. However, it is important to note that the PM-treatment does not allow us to distinguish between the thermal energy of the plume and the swept-up gas in contrast to the treatment by Arnold et al. [9]. Nevertheless, most of the important issues are comprised by the PM-model.

The dimensionless energies as a function of dimensionless time $\tau$ are shown in Fig. 2 for $\eta_{0}=40.8$ (which corresponds to the case also treated by Arnold et al. in his Figs. 2 and 3 [9]). The kinetic energy of the plume is initially constant for the nearly-free expansion and falls then gradually off, until the plume has stopped completely. The point at which the dimensionless plume front position $\xi=1$ in the PM-model, is where the plume mass is equal to the mass of the displaced background gas (see (3)-(4)). It is also in the center of the point-blast-wave regime [11], and is also exactly where the kinetic energy of the plume equals that of the swept-up gas. The kinetic energy of the plume decreases monotonically, as determined by the velocity of the front in the PM-model (see (12b)). Equations (12b) and (12c) also show that the two contributions of kinetic energy are always equal at $\xi=1$, independent of the specific target-gas interaction parameters, e.g. the initial front velocity $u_{0}$.

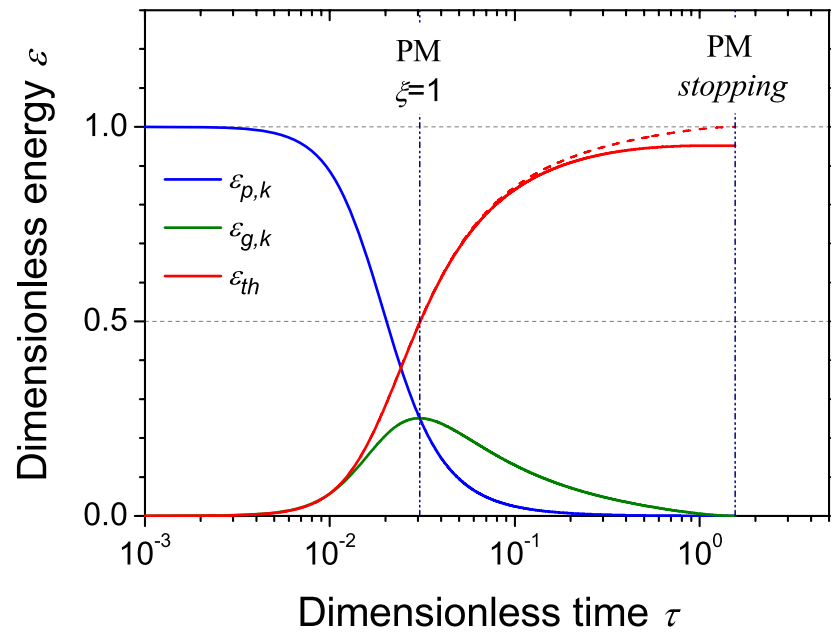

Fig. 2 The components of the dimensionless energy $\varepsilon$ (see (12) and (15)) as a function of the dimensionless time $\tau$ (see (3b)). $\varepsilon_{p k}$, the kinetic energy of the plume, (see (12b)), $\varepsilon_{g, \mathrm{k}}$, kinetic energy of the background gas (see (12c)), $\varepsilon_{\text {th }}$ excess thermal energy of the plume and the background gas (see (15)). The time for $\xi=1$ in the PM-model is indicated as well $\left(v_{f} / c_{g}=31.6\right.$ and $\gamma_{g}=5 / 3$ as in Ref. [9]) 
The thermal dimensionless (excess) energy is about $1 / 2$ of the total energy at $\xi=1$ (which can be seen from (15) since the last term on the right-hand side is of the order $2 / 1600=1 / 800$ ). It means that at $\xi=1$ approximately onehalf of the total energy is converted to thermal energy. At the stopping, nearly almost energy is thermal (which is also seen from (15) for $\eta=0$ ). The solid line includes the work made by the background gas, which means that the dimensionless thermal energy terminates at a point slightly below unity. Actually, if the work done by the external pressure is disregarded, the thermal dimensionless energy becomes exactly unity.

\section{Comparison with the model by Arnold et al.: the expansion}

The most comprehensive model for a plume expansion in a background gas, is the one by Arnold et al. who treat a specific case with a system of equations for the motion of the plume, an internal shock wave moving towards the center, an external shock wave moving in front of the contact surface and the background gas [9].

In order to compare the expansion dynamics from the PM-model we have changed the variables into those from the model of Arnold et al. for the specific system considered in Ref. [9]. The dimensionless time $\tau_{A}$ in Arnold's model is

$\tau_{A}=\sqrt{\frac{5}{3}}\left(\frac{2 \pi}{16} \frac{v_{f}^{2}}{c_{0}^{2}}\right)^{-\frac{1}{3}} \tau$

and the distance

$\xi_{A}=\left(\frac{2 \pi}{16} \frac{v_{f}^{2}}{c_{0}^{2}}\right)^{-\frac{1}{3}} \xi$

where $v_{f}$ is the initial expansion velocity in Arnold's model, both quantities with an exponent of $(-1 / 3)$.

The motion of the contact boundary for Arnold's system is shown together with the curve from the PM-model in Fig. 3. The contact boundary, i.e. the surface between the inner and outer shock region, is seen to collapse after the stopping, whereas the PM-model solely describes the slowing down to the end of the expansion. The overall agreement between the two models is surprisingly good, even though the PM-model is much simpler. The stopping distance and time are fairly similar, even though the PM-model overestimates the plume distance at the intermediate stage around $\xi=1$, since it does not include the loss of energy to the internal shock wave.

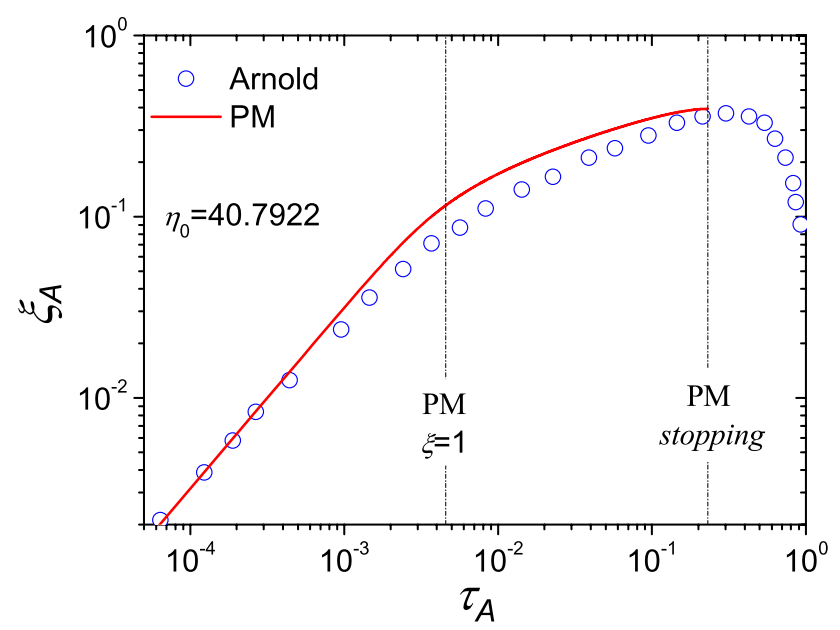

Fig. 3 Expansion distance $\xi_{A}$ as a function of time $\tau_{A}$ in the units from Ref. [9]. Parameters from Fig. 2. $\tau_{A}=0.149 \tau$ (see (16)), $\xi_{A}=0.115 \xi$ (see (17))

\section{Comparison with the model of Arnold et al.: energy balance}

The model of Arnold et al. [9] accounts for the detailed distribution of energy into the kinetic and thermal components of the plume as well as the background gas, whereas the PMmodel solely gives the total thermal energy of the plume and that of the background gas.

In Fig. 4 the kinetic energy of the plume (a), the total thermal energy (b) and the kinetic energy of the background gas (c) are shown from Arnold's model together with the results for the PM-model scaled to Arnold's time $\tau_{A}$. The kinetic energy of the plume calculated from the PM-model is very close to that from Arnold et al. calculated as the sum of the kinetic energy of the plume and the energy of the internal shock wave. The thermal energy from the PM-model is substantially higher than the thermal energy from the internal and external shock wave from Arnold's model in the final stage except around the stopping. In contrast, the kinetic energy of the background gas is lower in the PM-model than in Arnold's model. This deviation is caused by the generation of the external shockwave from thermal energy at the contact boundary, whereas all the swept-up mass in the PMmodel remains in the shell.

\section{Discussion}

The decisive feature in the PM-model is the compression of the plume mass and the swept-up background gas into an infinitesimally thin shell. This trick makes it possible to solve the hydrodynamic equations analytically, but it involves some problems. In principle, the interior behind the shell of the sphere is empty in the PM-model. In reality, 


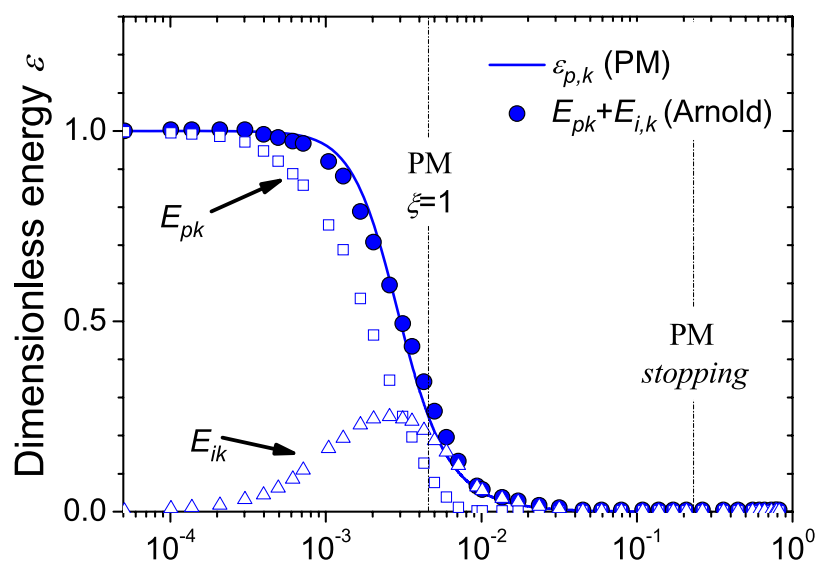

a
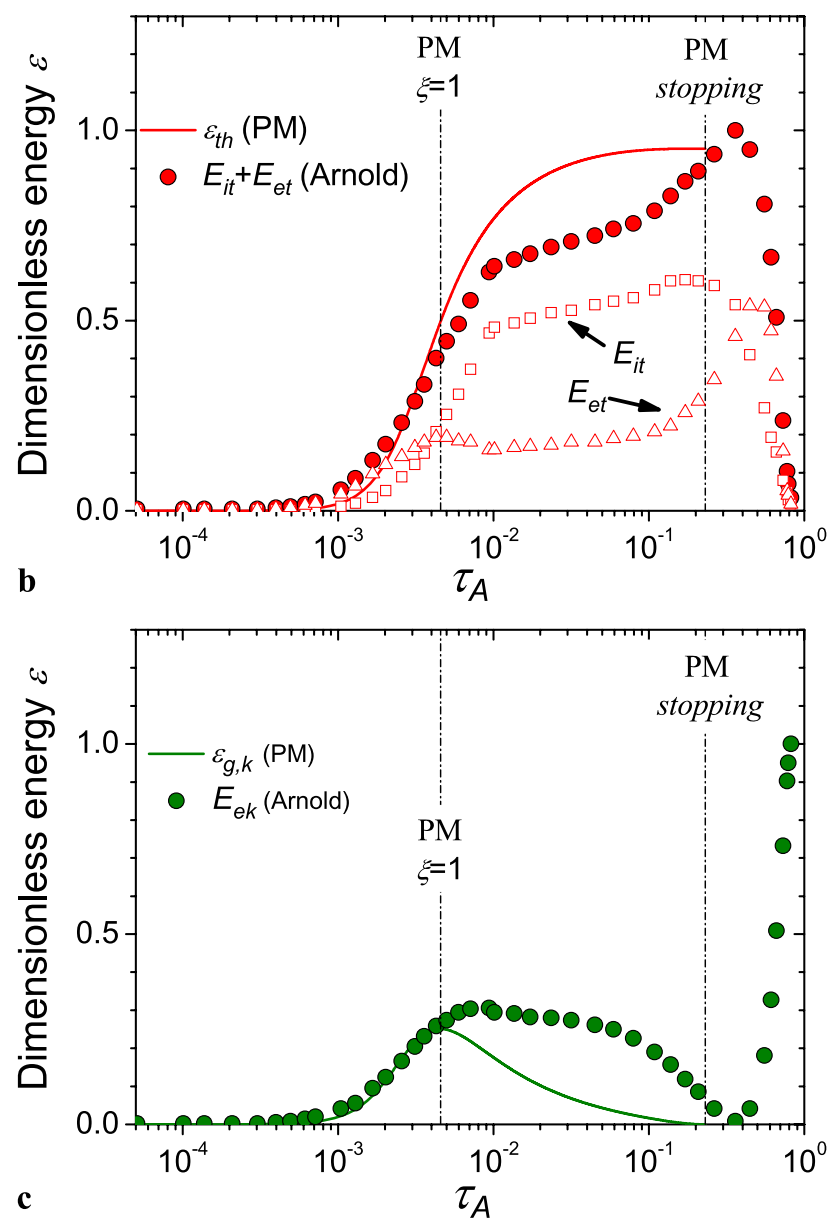

Fig. 4 Dimensionless energies with the same parameters as in Figs. 2 and 3. (a) Kinetic energy from the PM-model compared with the plume energy and the internal shock wave energy from Ref. [9]. (b) Thermal energy from the PM-model compared with thermal energy from the internal and external shockwave from Ref. [9]. (c) Kinetic energy of the background gas from the PM-model compared with the kinetic energy of the external shockwave

the space inside the sphere is dilute, but not empty [7, 9, 12]. However, a model with the plume mass distributed inside the shell becomes far more complex, and in view of the satisfactory agreement with the more complete model of Arnold et al. [9], there is hardly any reason to extend the PM-model.

Around and after the stopping of the plume the energy balance of the system becomes difficult to handle. It is not included in the PM-model, and also for other models the predictions are not accurate around the stopping distance [9, 16]. In the late stage, the diffusion of particles out of the plume volume becomes gradually more important $[4,5,7$, $11,17]$.

\section{Conclusion}

The energy balance of a plume expansion in a background gas can be described in a surprisingly simple way by the PM-model. The equations for the kinetic energy of the plume, the compressed background gas and the excess thermal energy of the system can be solved analytically and provide us with an instructive scheme for the plumebackground gas interactions. In the point-blast-wave regime around the dimensionless expansion radius $\xi=1$, the kinetic energy of the plume is exactly equal to kinetic energy of the swept-up background gas. At this point also the thermal energy of the system is approximately equal to the sum of the kinetic energy. With increasing time both components of kinetic energy become gradually converted into excess thermal energy of the plume and background gas.

Acknowledgements The authors thank Nikita Arnold for valuable discussions.

\section{References}

1. R. Eason (ed.), Pulsed Laser Deposition of Thin Films (Wiley, Hoboken, 2007)

2. C.R. Phipps (ed.), Laser Ablation and Its Applications (Springer, New York, 2007)

3. D.H. Lowndes, in Laser Ablation and Desorption, ed. by J.C. Miller, R.F. Haglund. Experimental Methods in the Physical Sciences, vol. 30 (Academic Press, New York, 1998), p. 475

4. J. Schou, Appl. Surf. Sci. 255, 5191 (2009)

5. M. Kuwata, B. Luk'yanchuk, T. Yabe, Jpn. J. Appl. Phys. 40, 4262 (2001)

6. S. Amoruso, R. Bruzzese, N. Spinelli, R. Velotta, M. Vitiello, X. Wang, Phys. Rev. B 67, 224503 (2003)

7. T.E. Itina, J. Hermann, P. Delaporte, M. Sentis, Phys. Rev. E 66, 066406 (2002)

8. R.F. Wood, K.R. Chen, J.N. Leboeuf, A.A. Puretzky, D.B. Geohegan, Phys. Rev. Lett. 79, 1571 (1997)

9. N. Arnold, J. Gruber, J. Heitz, Appl. Phys. A 69, S87 (1999)

10. M.R. Predtechensky, A.P. Mayorov, Appl. Supercond. 1, 2011 (1993)

11. S. Amoruso, J. Schou, J.G. Lunney, Appl. Phys. A 92, 907 (2008) 
12. A. Sambri, S. Amoruso, X. Wang, F. Miletto Granozio, R. Bruzzese, J. Appl. Phys. 104, 053304 (2008)

13. A. Sambri, M. Radovic', X. Wang, S. Amoruso, F. Miletto Granozio, R. Bruzzese, Appl. Surf. Sci. 254, 790 (2007)

14. A. Sambri, S. Amoruso, X. Wang, M. Radovic, F. Miletto Granozio, R. Bruzzese, Appl. Phys. Lett. 91, 151501 (2007)
15. S. Amoruso, R. Bruzzese, X. Wang, J. Xia, Appl. Phys. Lett. 92, $041503(2008)$

16. N. Huber, J. Gruber, N. Arnold, J. Heitz, D. Bäuerle, Europhys. Lett. 51, 674 (2000)

17. S. Amoruso, B. Toftmann, J. Schou, Phys. Rev. E 69, 056403 (2004) 